\title{
LEGISLATION
}

\section{Une décision récente de la Cour de Cassation sur la constitution d'une Chute d'eau (23 décembre 1924)}

\author{
Par Paul BOUGaULT, Avocal à la Cour d'Appel de Lyon.
}

\begin{abstract}
La Chambre civile de la Cour de Cassation vient de rendre un arrêt qui permel de préciser dune manière trés nette quelle est la sphère d'application des articles "vitaux: "de la loi du 16 octabre 1919 ; il oblige à lire avec attention deux: articles, ce qui est excellent, puisque tant de personnes ont, jusqu'à présent, donné leur avis sans les lire.
\end{abstract}

\section{Textes à invoquer pour constituer une chute.}

Tout entrepreneur d'un travail destiné à accumuler ou à produire de l'énergie, au moyen d'un lac ou d'un cours d'eau, doit se reporter à la loi du 16 octobre 1919, et se demander, avant de se mettre à l'veurre, quels sont les articles qu'il aura à invoquer.

C'est d'abord l'article $1^{\text {er }}$ : Nul ne peut disposer de l'énergic des marées, lacs et cours deau, sans une cession ou une autorisation de l'Etat "; ensuite, l'article 2 qui indique les chutes pour lesquelles une concession est nécessaire ; enfin les articles 4 et 5 : quels sont les droits que le concessionnaire peut invoquer et quelles procédures il peut entreprendre à l'encontre destiers?

Tous ces articles ont été lus, évidemment, par la "Société civile d'études » pour l'utilisation des eaux des lacs de SaintPoint et de Remoray (1). Ce titre seul nous indique que nous allons nous trouver en présence d'une entreprise un peu spéciale, très en dehors des dérivations banales, et dont le caractère distinctif sera constitué par l'accumulation des eaux, dans deux lacs préexistants, aux fins de régulariser le cours d'une rivière.

Le 17 août 1922, un décret était pris par le Présidenl de la République (voir le Journal Officiel du 25 août 1922, et la circulaire spéciale de la Chambre Syndicale des Forces hydrauliques, année 1922, n०376), " pour autoriser et déclarer d'ulilité publique " les travaux à entreprendre dans le département du Doubs. " suivant les dispositions d'un avant-projet présenté par la "Société demanderesse, en vue de la régularisation du cours " du Doubs, par la constitution d'une réserve de 14 millions " de mètres cubes d'eau dans les lacs de Saint-loint et de lie"moray ".

Selon la rubrique bien connue, le décret stipulait que la "présente déclaration d'utilité publique serait nulle et non avenue si les expropriations nécessaires pour l'exéculion des ouvrages n'étaient pas accomplies dans le délai de deux ans à parlir de

(1) Le barrage est prévu dans le lit du Doubs à environ $200 \mathrm{~m}$. du pont de Pallet. Le cahier des charges ne jrévoit pas la constitution d'une chute, ni l'ćtablissement d'une usine hydroélectrique, et encore moins la vente des kilowatts; l'entreprise est essentiellement destince à une accumulation d'eau. la date du décret". Enfin, la convention et le cahier des charges, acceptés d'un commun accord par le Ministre et la Sociélé, étaient approuvés.

La constitution d'une réserve de 11 millions de melres cubes dans un lac n'est pas accomplie sans que le plan d'eau soit surélevé considérablement, et il devenait nécessaire que le concessionnaire "occupat " les parcelles riveraines des lacs, car elles devaient ètre soumises à une submersion très fréquente, sinon continue.

Nous écrivons à dessein le mot "occuper " el nous n'hésitons pas à le souligner, pour plusieurs raisons.

D'abord c'est celui que la loi cmploie elle-mème, quand, dans son article 4, elle entend indiquer nettement le droit primordial du concessionnaire: : Si nous lisons cet article nous y voyons en première ligne les termes suivants : Pour l'exécution des travaux délinis au cahier des charges et régulièrement upprouvés par ladministration ainsi que par l'exploitation de la concession, le concessionnaire aura les droits suivants :- Occuper dans l'in" térieur du périmètre défini par l'acte de concession, les pro"priétés privées nécessaires à l'élablisscment des ouvrages do " retenue ou de prise d'eau, el des canaux d'adduction on de "fuite, lorsque cés canaux sont souterrains, ou s'ils sont à cicl "ouvert en se conformant à la loi du 29 avril 1845 ".

Ensuite, parce que ce lerme, grâce à sa signification très ample, et à l'acception tris large, qu'il recoit dans le langage juridique, a été choisi par le législateur, de préférence à tout autre, aussi, il n'est pas sans intérêt de précjser ce qu'il signifie: "L'occupation " ne commence pas par clre un droil, c'est un fait qui peut être accompli de diftérentes manières: on occupe un terrain aussi bien d'une facon provisoire que d'une façon définitive; cela dépend des intentions du concessionmaire, et des nécessités auxquelles il doit faire face : s’il pénc̀tre sur une parcelle pour établir un chantier, il y fera circuler des ouvriers, y déposera des matériaux; et lorsque l'ouvrage sera fini, il quittera la parcelle, la laissant peut-être fort endommagée, mais en tout cas complètement libre. Son occupation, en cela, n'aura été que provisoire.

Mais s'il édific, sur une partie de cette parcelle ou sur une autre, un barrage apjelé à subsister perpéluellement et en tout cas pendant les 75 ans de la concession, il occupe la parcelle, à litre définilif; il sait mêne, des l'origine de ses travaux, que 
son occupation aura ce caractère et, par conséquent, sera permenente a tilre de propriélć. Car si nous avons dans l'arsenal de nos lois un texte (celui du 11 juillet 1847) qui donne aux agricultcurs le droit d'appuyer l'exlrémité d'un barrage sur la rive opposée à celle qu'il s'agit d'irriguer, nous n'avions pas de loi comportant le droit "d'implantation du barrage "; et un pareil droit ne se comprendrait pas à titre de servilude.

Au contraire, à cé dernier litre, on peut créer un aqueduc d'irrigation en se conformant à la loi du 29 avril 1845 , c'està-dire que le propriétaire d'une terre et d'un droit d'eau séparés l'un de l'autre par des terrains intermédiaires, peut obtenir du tribunal le droit de placer sur ces surfaces des canaux à ciet ouvert; un aqueduc de peu d'importance ne pouvant ètre considéré comme une gêne considérable a été admis par la loi précitée à titre dé servitude; il est évident que le propriétaire du sol serait encore moins gêné par une canalisation placée dans le tréfonds, quand les travaux auront été faits dans les règles de l'art, et l'on peut concevoir, comme une servitude plutôt que comme une dépossession, la création chez un tiers d'un aqueduc souterrain.

Ces quelques notions suffisent à établir que l'on peut occuper une parcelle, à bien des titres différents : d'abord à titre provisoire, sans idée préconçue de s'y fixer ; ensuite à titre définitif, ce qui comporte deux aspects : l'occupation en qualitẻ de propriétaire, d'abord, quand il est impossible d'admettre la coexistence sur une même parcelle de deux propriétés : celle du sol et celle de l'ouvrage (par exemple, la propriété d'un barrage comporte la propriété du sol qui le soutient) et l'occupation à titre de servitude quand il n'est créé sur une parcelle qu'une simple gêne, canal à ciel ouvert, par exemple ou canal souterrain, simple dépossession d'un tréfonds, presque inaperçue à la surface.

Ajoutons, en dernier lieu, que l'on peut « submerger les berges . c'est-à-dire couvrir d'eau les parcelles dans lesquelles coule la rivière ou qui encadrent un lac; le mot berge correspond en effet à la partie plus ou moins profonde, mais toujours encaissée, dans laquelle se trouve le cours d'eau on le lac; cette submersion pourra aussi ètre permanente, au cas de reflux constant ou provisoire selon l'état des eaux (hautes, moyennes ou basses).

L'article 4 qui dans la loi du 16 octobre 1919 est relatif aux occupations est longuement 'rédigé ; c'est peut-être un inconvénient : mais grâce à cette dimension, l'article est complet et peut avoir la prétention très justifiée d'avoir prévu tous les cas possibles.

\section{Différentes occupations prévues par l'article 4 .}

Après avoir spécifié, dans les premiers alinéas ci-dessus rapportés, que l'occupation des terrains et la submersion des herges (ce qui est symonyme) constituent le droit de tout concessionnaire, par le fait de la concession elle-même et sans quil intervienne la moindre déclaration d'utilité publicque, l'article 4 cxempte de toute occupation les bâtiments, cours, jardins, attenant aux habitations; ensuite, l'article précise comment il comprend la mise en couvre par lc concessionnaire des droits qu'il lui confère : c'est ce qui fait l'objet des $6^{\mathrm{e}}, 7^{\mathrm{e}}, 8^{\mathrm{e}}$ et $9^{\mathrm{e}}$ alinéas.

A. Avant tout, puisque l'article entier est relatif seulement aux parcelles comprises dans le périmètre de la concession, il faut que le concessionnaire fasse circonscrire ce périmètre par l'autorité administrative : le préfet indique les parcelles à occuper, par un arrêté qui doil recevoir une publicité nécessaire pour que les propriétaires des parcelles puissent faire leurs observations. Le sixième alinéa est absolument formel : «L'exer-
" cice des droits conférés au concessionnaire par le présent article " est autorisé par arrêté préfectoral, pris après que les proprié"taires ont été mis à même de présenter leurs observations". Le règlement d'administration publique du 30 juillet 1920 qui indique les formalités à accomplir pour observer les prescriptions légales a minutieusement tracé les règles relatives à cet arrêté et à la publicité qu'il doit recevoir. Les tiers (déjà avisés, par l'enquête générale qui a été faite antérieurement au décret, de l'ampleur plus ou moins grande du projet), sont en qualité de propriétaires prévenus par une enquête dite parcellaire, ordonnée par l'article 26 : le plan des propriétés frappées de ces servitudes reste déposé pendant huit jours à la mairie de la commune où les propriétés sont situées; ce dépôt est annoncé par la publicité ordinaire, et une notification directe des travaux projetés est en outre donnée par la voie administrative aux intéressés.

B. Ces démarches étant faites, l'occupation peut commencer immédiatement; pour combien de temps? Alors même que dans l'esprit du concessionnaire elle ne devrait ètre que provisoire, il se peut qu'elle se prolonge longtemps, plus longtemps même que la durée prévue au cahier des charges, pour l'exécution des travaux. Elle peut aussi avoir rendu les terrains impropres à l'agriculture; il est de toute justice que le propriétaire, dépossédé pendant plus longtemps qu'il n'était prévu, puisse exiger du concessionnaire l'acquisition du sol, non seulement dans la partie où celui-ci est déprécié, mais encore dans toute l'étendue de la parcelle. Si, au contraire, l'occupation ayant pris fin n'a causé qu'un dommage temporaire comme elle, le propriétaire ne doit demander que des dommages-intérêts. Le $7^{\mathrm{e}}$ alinéa règle cette situation : "Lorsque l'occupation ainsi faite prive le propriétaire " de la jouissance du sol pendant une durée supérieure à celle " prévue par le cahier des charges pour l'exécution des travaux, " ou, lorsqu'après cette exécution les terrains ne sont plus pro" pres à la culture, le propriétaire peut exiger du concessionnaire " l'acquisition du sol. La pièce de terre trop endommagée ou "trop dépréciée doit être achetée en totalité si le propriétaire "l'exige ".

Comme on le voit, rien ne dit explicitement dans cet alinéa que l'indemnité doit être versée préalablement à l'occupation; implicitement même le contraire ressort clairement du texte car comment donnerait-on une indemnité pour dommage, sans connaître l'importance de ce dommage, ce qui suppose qu'il est accompli ? (1) Comment convertirait-on cette indemnité en un prix d'acquisition, à la demande du propriétaire, avant que l'on sache si ce dernier se trouvera dans les cas où il peut l'exiger (trop grande durée des travaux, détérioration importante). Aussi toutes les indemnités ou prix de cession seront-ils réglés à la fin de l'occupation, et au cas où les parties ne seraient pas d'accord, par le tribunal civil, proclamé compétent pour tout ce qui regarde ces deux questions, par le se alinéa : "Les indem" nités auxquelles pourra domner lieu l'application du présent. " article, ainsi que les contestations qu'il soulèvera seront "réglées par la juridiction civile; il sera procédé devant ces " tribunaux comme en matière sommaire, et s'il y a lieu à exper" tise, il pourra n’ètre nommé qu'un seul expert ".

(1) On peut même dire que l'article $t$ précise explicitement qı e l'indemnité ne peut pas être donnée próalablement pour une occupation susceptible d'ctre provisoire; car le dernier alinéa stipule que le versement préalable ne sera dù que pour une oceupation dont le caractère permanent est justifié dès le début; a contrario, il n'est exigé aucun versement próalable dans les autres cas. Voir ci-dessous lettre $C$. 
C. Dans un cas néanmoins, lindemnile doit itre prealable; c'est la conséquence du mème principe appliqué en sens inverse. Ce cas est celui-ci : dès le début, il est certain que loceupation considérée ne pourra ètre que permanente el définitive; par exemple : le barrage, qui est un mur solide (il faut au moins l'espérer), ou par la submersion constante qui sera due à la surélération du plan deau, mème en eaux basses ou moyennes; ou enfin les canalisations d'amenée ou de fuite. Dans ces cas, la valeur d'acquisition où l'indemnité sera fixée par la juridiction civile, avec ou sans expertise suivant que les juges trouveront ou ne trouveront pas dans la cause des éléments dappréciation. Il est juste qu'en principe le concessionnaire ne puisse sinstaller qu'après un paiement; seulement, comme il pourra ètre victime d'une trop grande lenteur, la loi lui donne en tout temps le droit d'employer une procédure dite d'urgence, qui est prévue par les articles 66 et suivants jusquà 74 de la loi du 3 mai 1811 ; sans nous éterniser sur ce point, disons que cette procédure consiste essentiellement dans la consignation d'une somme fixée par le tribunal et versée à titre de provision avant la prise de possession. Mais la juridiction civile a seule compétence pour fixer l'indemnité définitive : Tout ce qui est relatif à l'occupation permanente est réglé par le dernier alinéa de l'article, 'alinéa ainsi conçu : "Lorsque l'occupation ou la dépos" session devra ètre permanente, l'indemnité sera préalable. "Toutefois si l'urgence des travaux est reconnue par arrèté " préfectoral, cet arrêté et l'arrêté déclaratif des droits seraient " notifiés et l'indemnité sera réglée dans les formes prévues par " les articles 66 à 74 de la loi du 3 mai 1841, la juridiction civile " restant compétente pour la fixation définitive de cette indemnité "

En une phrase, résumons toute cette analyse et constatons que la juridiction civile garde dans tous les cas pleine compétence en matière d'indemnité pour occupation, soit que cette indemnité constitne la simple compensation d'un dommage disparu, soit qu'au contraire elle se transforme, sur la demande justifiée du propriétaire, en un prix d'acquisition : dans ces deux cas, elle n'est fixée qu'à la fin du délai imparti au concessionnaire pour terminer les travaux. Cette indemnité sera au contraire fixée préalablement à l'occupation, au cas où il sera d'ores et déjà constant qu'elle sera permanente.

\section{Application de ces principes par l'arrêt de la Cour de Cassation.}

Conformément à ces principes, la Société civile d'Etudes, pour l'utilisation des eaux des lacs de Saint-Point et de Remoray, après avoir obtenu le décret précité du 17 août 1922 (1), se préoccupe d'obtenir une situation régulière, tant au point de vue administratif qu'au point de vue des tiers.

Au point de vue administralif, le préfet du Doubs prit, le $1^{\text {er }}$ juin 1923, un arrêté déterminant les parcelles à occuper pour l'établissement des servitudes prévues à l'article 4, paragraphes 1 et 2 de la loi du 16 octobre 1919, et autorisa la Société tant à occuper jusqu'à l'expiration de la concession les terrains désignés à l'état parcellaire, qu'à y exercer les droits dẻfinis par l'article 4, paragraphes 1 et 2 précités.

(1) L'accumulation projetéc par l'entreprise a comme but principal la régularisation du cours du Doulss. Cotte rivière, pen après sa source situce sur la commune de Mouthe, dans l'arrondissement de Pontarlier. entre dans le lac de Saint-Point, qui, séparé du lac de Remoray par des prairies moullées, couvre une superficie de 160 hectares, sur une longueur de 6 kilometres environ et une largeur de 600 mètres en moyenne.
Alu poinl de nue des propriétaires intéressés, la Sociélé adressa ¿̀ chacun des détenteurs des 180 parcelles désignées une lettre circulaire, en clate du '29 décembre 1923 , faisant des offres d'achat du Lerrain ou de conslitution de servitude; elle indiquait les deux causes juridiques, car le propriétaire, étant donné que l'occupation pouvait etre permanente, avait le droit d'exiger l'acquisition totale.

La grande majorité des propriétaires (101) refusa les offres.

Sans doute, devant le décret de concession ils ne pouvaient pas s'opposer à ce que la Société occupât les parcelles nécessaires, mais ils prétendaient que la déclaration d'utilité publique leur donnait le droit de requérir, pour la fixation de l'indemnité, la réunion d'un jury d'expropriation. A cette fin, ils préparèrent une requete dans laquelle ils multipliaient les citalions de textes, demandant qu'il leur fut domé acte de leur consentement à céder "leurs propriétés frappées dans leurs parties par le décret "d'occupation provisoire à la Société du Lac de Saint-Point, " mais qu'il plût au tribunal de désigner 'en 'conformité de " l'article 55 de la loi du 3 mai 1841, de l'article 9 de la loi du " 29 décembre 1892 et de l'article 14 des lois du 3 mai 1841, " 6 novembre 1918 et 17 juillet 1921 , le jury spécial d'expro" priation chargé de fixer les indemnités à eux dues ainsi que "le magistrat directeur".

La requête, très habilement, ne cite mème pas la loi du 16 octobre 1919; elle ne tendait pas à une assignation devant le tribunal civil, mais réclamait la convocation du jury.

Les juges ordonnèrent ce qui leur était demandé, désignèrent par jugement du 15 mai 1924, dont le sommaire est donné par la Gazette des Tribunaux du 7 janvier 1925, le magistrat directeur et les membres du jury ; ils affirmèrent qu'ils agissaient dinsi en vertu de la loi du 3 mai 1841, - - ce qui n'a rien d'étonnant - mais aussi, et cela est profondément étonnant, en vertu de l'article 4 de la loi du 16 octobre 1919.

Voici d'ailleurs le texte de leur jugement rendu entre la Société et un grand nombre de demandeurs parmi lesquels la commune de Labergement-Ste-Marie :

"Ouï le Ministère public, en ses conclusions, M. Roux, juge commis, en son rapport, vu la requête présentée par $M^{e}$ Louvier, avoué, avec le dossier joint et les pièces y annexées, vu la loí du 3 mai 1841 modifiée et complétée par les lois des 21 avril 1914, 6 novembre 1918 et 17 juillet 1921 , notamment les articles 14 , 29 , 30 et 55 ; vu la loi du 29 novembre 1892 et spécialement l'article 9; vu le décret du 17 aoûl 1922, constituant la Société civile d'Etudes, dont le siège social est à Montbéliard; concessionnaire, etc., etc.; vu l'arrèté de $\mathrm{M}$. le préfet du Doubs en date du 1 er juin 1923 déterminant les parcelles à occuper pour l'établissement des servitudes prévues à l'article $4 \$ 1^{\circ}$ et $2^{\circ}$ de la loi du 16 octobre 1919; wu le dil article 4 de la loi du 16 octobre 1919 ; vu la liste des jurys d'expropriation pour l'année 1924 , dressée par le Conseil général du département du Doubs, au cours de sa session de 1923.

"Attendu que des pièces versées aux débats, il est constant que depuis tout au moins le 29 mars 1918, date de l'arrèté de M. le Préfet du I)oubs, autorisant l'occupation temporaire des terrains, propriétés des réclamanls ; que, depuis cette époque, la Société sus-nommée, à défaut d'accord avec les intéressés, n'a fait aucune diligence pour faire fixer légalement l'indemnité d'expropriation.

"Attendu que tous les requéranls, en leur qualité de propriétaires à exproprier, déclarent consentir à la cession, mais sont seulement en désaccord sur le prix. 
"Qu'il y a donc lieu de faire cn l'état l'application de l'artıcle 14 in fine de la loi du 3 mai 1841, modifié par la loi du 6 novembre 1918 (1) ainsi que l'article 9 de la loi du 9 décembre 1892; attendu que la procédure est régulière et bien fondée.

"Par ces motifs, le tribunal, vu la requête, les pièces jointes, les textes de lois, décrets et arrêtés sus-visés, ouï M. le Procurcur de la République en ses réquisitions, et M. Roux, juge commis en son rapport, après en avoir délibéré, dit recevable et mal fondée la requête des concluants; en conséquence leur donne acte du consentement par eux donné de la cession à la Sociélé civile d'Etudes pour l'utilisation des eaux des lacs de St-Point et de Remoray de leurs propriétés frappées par l'arrêté d'occupation de M. le Préfet du Doubs, du 1er juin 1923.

"Commet M. Roux, et à son défaut M. Panatard, juges suppléants pour remplir les fonctions attribuées par la loi du 3 mai 1841 au magistrat directeur du jury chargé de fixer l'indemnité, dit qu'en cas d'empêchement, il sera procédé à ieur remplacement par ordonnance de M. le Président rendue sur simple requête présentée par la partie la plus diligente,

"Désigne, après choix fait en chambre du Conseil et sur la liste à ce dressée par le Conseil général du Doubs, et pour former la liste de session du jury spécial chargé de fixer définitivement le montant des indemnités, .MM. N..., etc., dit que les frais de la présente instance seront, quant à ce, à la charge des demandeurs, pour être ultérieurement compris dans la masse des frais d'expropriation et répartis définitivement en conformité de la décision du jury. Ainsi fait et jugé ».

\section{Pourvoi et moyens du pourvoi.}

Devant la Cour de Cassation, la Société soutient que la loi du 16 octobre 1919 attribue normalement, non pas au jury, mais au tribunal civil, une compétence stricte, et exclusive pour évaluer l'indemnité en raison de toute occupation des parcelles comprises dans le périmètre de la concession, aussi bien quand cette occupation est provisoire que quand elle est définitive et permanente, quand elle donne lieu à une indemnité de dépossession ou à un prix de cession de terrain, ou à un prix d'établissement de servitude.

Il semblerait vraiment qu'il n'y eut pas de place pour la discussion, quand on a suivi les faits accomplis et les précautions prises par la Société, et quand on les a mis en parallèle, comme nous l'avons fait, avec les différents paragraphes de l'article 4.

Ce qu'il y a de plus frappant, c'est qu'un jugement ait ordomné une expropriation, en s'appuyant précisément sur un article 4 de fla loi du 16 octobre 1919 qui, est muet sur l'expropriation.

Ce n'est pas que la procédure expropriative soit en elle-mème incompatible avec la loi du 16 octobre 1919, on ne saurait le prétendre puisque précisément l'article 5 prévoit que l'entrepreneur de la chute pourra y recourir, mais l'article 5 stipule

(1) Cet article 14 se termine ainsi : "Dans le cas où les propriétaires à exproprier consentiraient à la cession, mais où il n'y aurait point d'accord sur le prix, le tribunal domnera acte du consentement et désignera le magistrat directeur du jury, sans qu'il soit besoin de rendre le jugement d'expropriation ni de s'assurer que les formalités prescrites par le titre II ont été remplies ". Quant à l'article 55 qui est cité plus haut, il est relatif i la possibilité pour les parties de demander la convocation du jury si dans les six mois du jugement d'expropriation, l'arministration ne poursuit pas la fixation de l'indemnité. expressément d'abord qu'il s'applique à une sphère absolument étrangère à l'article 4 , et qu'ensuite il entend ne rien innover à ce qui a été spécifié à cet article 4 . Si un texte ne prête pas à l'équivoque, c'est bien certainement l'article 5 : "Lorsque l'aménagement de l'entreprise nécessite l'occupation définitive de propriétés privées dans des cas autres que ceux prévus par l'article 4, l'utilité publique de l'entreprise peut, si l'intérêt économique de la nation le justifie, être déclarée par l'acte qui approuve la concession... Lorsque l'utilité publique a été déclarée, s'il y a lieu à expropriation, il est procédé conformément à la loi du 3 mai 1841, sans qu'il soit en rien dérogé aux dispositions des articles 4 el 6 ."

Pourrait-on dire d'une façon plus claire que la déclaration d'utilité publique n'entraîne pas fatalement l'expropriation et qu'elle laisse subsister complètement la compétence du tribunal civil, prévue à l'article 4 pour les indemnités de simple occupation,

Il est facile de trouver d'ailleurs des cas où l'article 5, indépendamment de l'article 4, est le seul pouvant être mis en cuvre. On pourrait dire que cet article 5 est fait pour suppléer aux défaillances de celui qui le précèdle, et qui n'excède pas les limites dans lesquelles il est renfermé par son propre texte.

On peut citer les exemples si fréquents que voici :

10 L'article 4 ne peut s'appliquer aux bâtiments, cours et. jardins attenant aux habitations; si donc la surélévation du plan d'eau vient à mouiller un bâtiment, celui-ci doit ètre acheté en entier, soit à l'amiable, soit par expropriation.

$2^{\circ}$ L'article 4, - limité à la submersion des berges et à l'occupation nécessaire à un ouvrage de prise et aux canaux d'adduction et de fuite, - est muet au sujet du terrain qui doit recevoir l'usine. Le législateur a pensé que le terrain convenant à ce genre de construction n'était pas limité à un petit nombre de parcelles comme pour le choix d'un barrage, qui ne peut ètre fait qu'aux endroits d'étranglement de la vallée; l'entrepreneur est censé le choisir librement. Mais s'il était avéré qu'il s'est heurté pratiquement à des difficultés insurmontables dans ses pourparlers, le jury d'expropriation devrait être convoqué, après la déclaration d'utilité publique prononcée à l'égard de la parcelle nécessaire à l'établissement industriel.

\section{Analyse de l'arrêt de la Chambre civile.}

L'arrêt est très bref', mais très précis. Après avoir reproduit, dans son entier, l'ărticle 4 de la loi du 16 octobre 1919, il constate d'abord que sa signification est la suivante : Lorsque le concessionnaire a usé des droits d'occupation et de submersion que lui confère cet article, et lorsque les propriétaires exigent l'acquisition du sol, la juridiction civile statuant en matière sommaire, doit se prononcer sur le prix ; d'autre part, l'article 5 ajoute que si l'occupation des parcelles dans des cas autres que ceux prévus par l'article 4 est nécessaire, il sera procédé conformément à la loi du 3 mai 1841 ; or, par l'arrêté en date du $1^{\mathrm{er}}$ juin 1923 , le préfet avait autorisé ${ }^{2}$ exercice des droits conférés au concessionnaire par l'article 4 , paragraphes 1 et 2 de la loi, et désigné les terrains pouvant être occupés; enfin le jugement en concordance absolue avec l'arrêté administratif s'était rapporté à l'article 4.

" Il ne peut donc résulter, dit la Cour suprème, ni expres" sément, ni implicitement de ses énonciations qu'il s'agissait "d'un aménagement de l'entreprise nécessitant, ainsi que le 
"prévoit l'article 5 de la mème loi, l'occupation définitive de " propriétés privées dans des cas autres que ceux prévus par "larticle 4; des lors, en renvoyant les parties devant le jury "spécial d'expropriation, pour ètre procédé conformément à "la loi du 3 mai 1841, le jugement attaqué a violé le texte sus"visé, par conséquent le jugement est cassé, au préjudice de la "commune de Labergement-Sainte-Murie et autres qui l'avaient "obtenu ".

\section{Texte de l'arrêt.}

Ouï à l'audience de ce jour, M. le conseiller Davaine, en son rapport, MM. Frénoy et Talamon, avocats des parties, en leurs observations respectives, ainsi que M. Langlois, avocat général, en ses conclusions, et après en avoir délibéré conformément à la loi.

Donne défaut contre Girard, Ragnin, etc., les communes des Granges-Sainte-Marie, de Malbuisson, de Saint-Point, des Grangettes, d'Oge, de Montperroux, de Remoray, contre dame Pielle, contre le Préfet du Doubs.

Sur le premier moven, vu l'article 4 de la loi du 16 octobre 1919 :

"Pour l'exécution des travaux définis au cahier des charges et régulièrement approuvés par l'Administration, le concessionnaire a le droit d'exercer les servitudes suivantes :

“10 Occuper dans l'intérieur du périmètre défini par l'acte de concession, les propriétés privées nécessaires à l'établissement. des ouvrages de retenue ou de prise d'eau, et des canaux d'adduction ou de fuite lorsque ces canaux sont souterrains ou s'ils sont à ciel ouvert, en se conformant à la loi du 29 avril 1845 ;

“ $2^{\circ}$ Submerger les bergès, par le relèvement du plan d'eau ;

“30 S’il s'agit d'une usine de plus de 10.000 kilowatts, occuper temporairement tous terrains et extraire tous matériaux nécessaires à l'exécution des travaux, en se conformant aux prescriptions de la loi du 29 décembre 1892.

«Sont exemptés de ces servitudes les bâtiments, cours et jardins attenant aux habitations.

"L'exercice des droits conférés au concessionnaire par le présent article est autorisé par arrêté préfectoral pris après que les propriétaires ont été mis à mème de présenter leurs observations.

"Lorsque l'occupation ainsi faite prive le propriétaire de la jouissance du sol pendant une durée supérieure à celle prévue par le cahier des charges pour l'exécution des travaux ou lorsque, après cette exécution, les terrains ne sont plus propres à la culture, le propriétaire peut exiger du concessionnaire l'acquisition du sol. La pièce de terre trop endommagée ou trop dépréciée doit être achetée en totalité si le propriétaire l'exige.

"Les indemnités auxquelles pourra donner lieu l'application du présent article ainsi que les contestations qu'il soulèvera seront réglées par la juridiction civile. Il sera procédé devant, les tribunaux comme en matière sommaire et, s'il y a lieu à expertise, il pourra n'être ncmmé qu'un seul expert.

"Lorsque l'occupation ou la dépossession devra être permanente, l'indemnité sera préalable. Toutefois, si l'urgence des travaux est reconnue par arrèté préfectoral, cet arrêté et l'arrêté déclaratif des servitudes seront notifiés et l'indemnité sera réglée dàns les formes prévues par les articles 60 à 74 de la loi du 3 mai 1841, la juridiction civile restant compétente pour la fixation définitive de cette indemnité. "
Attendu qu'il résulte de cette disposilion que, lorsque le concessionnaire ayant usé des droits d'occupation et de submersion que lui confère cet article, les propriélaires exigent de lui l'acquisition du sol, les indemnités et les contestations soulevées pour l'application de ce texte sont réglées par la juridiction civile comme en matière sommaire; mais que l'article 5 de la même loi ajoute que si l'aménagement de l'entreprise nécessite l'occupation définitive des propriétés privées dans des cas autres que ceux prévus par l'article 4, l'utilité publique peut ètre déclarée par l'acte qui approuve la concession, et, s'il y a lieu à expropriation, il est procédé conformément à la loi du 3 mai 1841 .

Attendu que, par décret du 17 août 1922 la Société civile d'Etudes pour l'utilisation des eaux des lacs de Saint-Point et de Remoray a obtenu la concession des travaux destinés à la régularisation du cours du Doubs ct déclarée d'utilité publique par le même décret.

Que, par arrèté du 1er juin 1923, le Préfet du 1)oubs a autorisé l'exercice des droits conférés au concessionnaire par les paragraphes 1 et 2 de l'article + précité, et désigné les terrains pouvant être occupés.

Attendu que les propriétaires de ces terrains ont consenti à leur cession et que l'accord n'ayant pu se faire sur l'indemnité ils ont présenté requète au tribunal civil de Pontarliel, aux fins de désignation du jury spécial chargé de fixer les indemnités, conformément à la loi du 3 mai 1841 .

Que le tribunal a fait droit à cette requête.

Attendu que, d'après le moyen, le jugement attaqué aurait violé l'article 4 de la loi du 16 octobre 1919 , le tribunal civil lui-même, et non le jury d'expropriation étant compétent pour régler le prix des cessions amiables dont s'agit.

Attendu que le jugement vise, en effet, uniquement l'article 4 de la loi ;

Qu'il ne résulte ni expressément, ni implicitement de ses énonciations qu'il s'agissait d'un aménagement de l'entreprise nécessitant, ainsi que le prévoit l'article 5 de la même loi, l'occupation définitive des propriétés privées dans des cas autres que ceux prévus à l'article 4 .

Que, dès lors, en renvoyant les parties devant le jury spécial d'expropriation, pour être procédé conformément à la loi du 3 mai 1841, le jugement attaqué a violé le texte sus-visé.

Par ces motifs, et sans qu il y ait lieu de statuer sur le deuxième moyen, casse et annule le jugement rendu entre les parties par le tribunal civil de Pontarlier le 15 mai 1921, renvoie devant: le tribunal civil de Besançon.

\section{Explication complémentaire.}

Nous ne voudrions pas insister encore sur ce fait que nous croyons avoir précisé d'une façon suffisante : l'article 4 domne compétence au tribunal civil pour statuer sur toutes les indemnités à titre d'occupations provisoires on définitives, à la condition qu'il s'agisse de parcelles comprises dans le périmètre de la concession, et d'occupations susceptibles d'être faites par un concessionnaire nanti d'un simple décret. Nous croyons loutefois nécessaire de souligner une différence entre l'occupation provisoire ou temporaire envisagée par l'article 4 de la loi du 16 octobre 1919 et une autre occupation désignée sous les mèmes 
noms, ce qui est prévuc par une loi bien antérieure, celle du 29 décembre 1892.

La différence est grande.

La loi du 29 décembre 1892 est une loi générale qui autorise l'entrepreneur de tout travail public, à occuper toute parcelle, sauf celles qui sont closes el attenantes aux habitations, à la condition d'y être autorisé par un arrêté préfectoral, affiché dans la commune; cette occupation peut avoir lieu, soit pour faire de simples travaux de nivellement et de mesure, soit jour ramasser et même extraire des matériaux que contient la parcelle. Comme le fait remarquer M. Hauriou, édition de 1921, page 735 , cette loi est extrêmement large : l'arrêté préfectoral peut donner l'autorisation à tout agent de l'administration, que ce soit un entrepreneur, que ce soit un concessionnaire, que ce soit un fournisseur de matériaux.

Par quelle aberration le législateur du 16 octobre 1919 a-t-il, dans l'article 4 , limité aux ouvrages nécessaires à une chute de plus de 10.000 kilowatts, le droit d'occupation temporaire : ce que est certain, c'est que le $3^{\circ}$ de cet article existe et qu'il est ainsi conçu : "S'il s'agit d'une usine de plus de 10.000 kilowatts, le concessionnaire a le droit d'occuper temporairement tous terrains et extraire tous matériaux nécessaires à l'exécution des travaux en se conformant aux prescriptions de la loi du 29 décembre 1892 "; après avoir remarqué que cet. inexplicable alinéa refuse à une chute de 9.000 kilowatts le droit que peut avoir tout entrepreneur pour faire un chemin vicinal des plus modestes, il importe encore plus d'observer la sphère de cet alinéa, qui n'est pas du tout semblable à la sphère des autres : en effet, tous les autres que nous avons eu soin de reproduire, envisagent les occupations comprises dans le périmètre de la concession, et en confie le contentieux au tribunal civil : à ce litre et dans cette limite, ils se suffisent à eux-mèmes et sont indiscutables.

Pour les parcelles qui sont el resteront en dehors de la concession, il aurait été infiniment commode de pouvoir recourir à l'occupation temporaire prévue par la loi du 29 décembre 1892, puisque l'article 4 de la loi du 16 octobre 1919 ne s'applique plus. Nous avons senti dernièrement tout le préjudice que cause aux hydrauliseurs dont la chute n'a pas 10.000 kilowatts, l'inexplicable alinéa. Une parcelle très éloignée du périmètre de la concession devait être utilisée, pendant la construction, pour l'établissement essentiellement provisoire d'une galerie d'évacuation. Il aurait fallu y renoncer, si la chute n'avait pas été déclarée d'utilité publique. L'expropriation devenait donc possible, non pas ne vertu de l'article 4 , mais en vertu de l'article 5 qui est général et s'applique à toutes les parcelles nécessaires et une acquisition totale a réparé l'impossibilité d'une occupation provisoire.

Si jamais la loi devait être corrigée, le $3^{\circ}$ de l'énumération contenue dans l'article 4 sérait le premier à disparaître. Par le fait qu'il existe il ne permettrait même pas à un entrepreneur de faire des travaux de mesure et de nivellement sur une propriété privée.

Heureusement, sur ce dernier point, l'article 28 de la loi a permis de tourner la difficulté : il y est dit que des "règlements " d'administration publique détermineront les conditions de la " loi et fixeront notamment .10 les conditions dans lesquelles " les propriétaires seront tenus de laisser faire sur leurs pro"priétés tous travaux de mensuration ou de nivellement". C'est en vertu de ce texte qu'a été pris, dans des termes d'ailleurs fort simples, le décret du 20 janvier 1923, Journal Officiel du 24 janvier. 